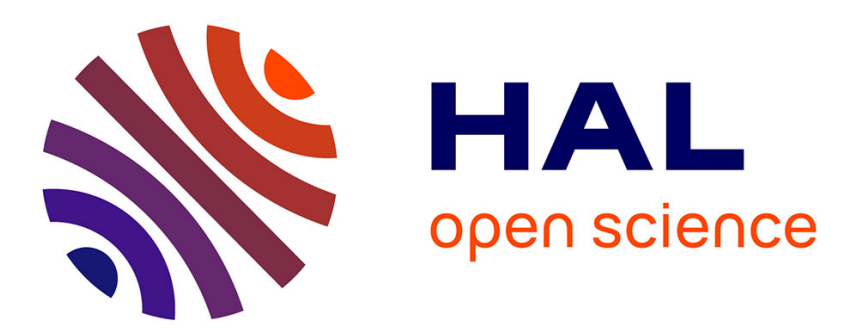

\title{
Event-driven simulations of nonlinear integrate-and-fire neurons
}

\author{
Arnaud Tonnelier, Hana Belmabrouk, Dominique Martinez
}

\section{To cite this version:}

Arnaud Tonnelier, Hana Belmabrouk, Dominique Martinez. Event-driven simulations of nonlinear integrate-and-fire neurons. Neural Computation, 2007, 19 (12), pp.3226-3238. 10.1162/neco.2007.19.12.3226 . inria-00103500

\section{HAL Id: inria-00103500 https://hal.inria.fr/inria-00103500}

Submitted on 18 Oct 2007

HAL is a multi-disciplinary open access archive for the deposit and dissemination of scientific research documents, whether they are published or not. The documents may come from teaching and research institutions in France or abroad, or from public or private research centers.
L'archive ouverte pluridisciplinaire HAL, est destinée au dépôt et à la diffusion de documents scientifiques de niveau recherche, publiés ou non, émanant des établissements d'enseignement et de recherche français ou étrangers, des laboratoires publics ou privés. 


\title{
Event-driven simulations of nonlinear integrate-and-fire neurons
}

\author{
A. Tonnelier, H. Belmabrouk, D. Martinez \\ Cortex Project, LORIA, Campus Scientifique, B.P. 239, 54506 \\ Vandoeuvre-lès-Nancy, France
}

\begin{abstract}
Event-driven strategies have been used to simulate spiking neural networks exactly. Previou work is limited to linear integrate-and-fire neurons. In this note we extend event driven schemes to a class of nonlinear integrate-and-fire models. Results are presented for the quadratic integrate-and-fire model with instantaneous or exponential synaptic currents. Extensions to conductance-based currents and exponential integrate-and-fire neurons are discussed.
\end{abstract}

\section{Introduction}

Our current theoretical understanding of the properties of neural systems is mainly based on numerical simulations from single cell models to neural networks. Recent experimental evidence has accumulated which suggests that precise spike-time coding is used in various neuronal systems (VanRullen et al., 2005). Individual spikes can be highly reliable and precisely timed with a submillisecond accuracy (Berry et al., 1997; Mainen and Sejnowski, 1995). Synaptic plasticity depends critically on the relative timing of pre- and postsynaptic spikes; a synapse is strengthened if the presynaptic spike occurs shortly before the postsynaptic neuron fires, and the synapse is weakened if the sequence of spikes is reversed (Bi and Poo, 1998). It is therefore important to have accurate numerical schemes to calculate spike times.

Integrate-and-fire neurons reproduce many features of the neuronal dynamics (Izhikevich, 2003; Tonnelier and Gerstner, 2003; Gerstner and Kistler, 2002) and are widely used in the numerical simulations of spiking neural networks. Two strategies have been used for the simulation of integrate-and-fire neural networks: time-stepping methods that approximate the membrane voltage of neurons on a discretized time and event-driven schemes where the timings of spikes are calculated exactly. By definition time-stepping approximations are imprecise and it has been shown that time steps have to be chosen correctly to reproduce the synchronization properties of networks of spiking neurons (Hansel et al., 1998). Standard time-stepping algorithms (Euler, Runge-Kutta) have to be modified to give an accurate approximation of firing times (Shelley and Tao, 2001). A fundamental limitation on the accuracy of any time-stepping methods is imposed by the smoothness of the postsynaptic potentials (Shelley and 
Tao, 2001). High-order time-stepping algorithms can be constructed only if the onset of postsynaptc conductance changes has smooth derivatives. Exact simulations avoid these problems. The term exact method or exact simulation means that spike timings are analytically given or are derived from the analytical formulation of the membrane potential. Thus it is possible to have an arbitrary precision (up to the machine precision) of spike timings. This method has become increasingly popular (Mattia and Giudice, 2000; Makino, 2003; Rochel and Martinez, 2003; Brette, 2006; Rudolph and Destexhe, 2006) but it applies to a limited class of neuron models, mainly the linear integrate-and-fire models. It is known that the leaky integrate-and-fire model has some limitations: it has an unrealistic behavior close to the threshold and reproduces only some characteristics of conductance based neuron (Fourcaud-Trocmé et al., 2003). More realistic models include nonlinear spike-generating currents that allow replacement of the strict voltage threshold by a smooth spike initiation zone. Here we simulate exactly the quadratic integrate-and-fire neuron (Ermentrout and Kopell, 1986). In the quadratic integrate-and-fire (QIF) model, the membrane potential follows

$$
C \frac{d V}{d t}=q\left(V-V_{t h}\right)^{2}-I_{t h}+I_{s}(t),
$$

where $V$ is the membrane voltage, $C$ is the membrane capacitance, $q$ is a parameter characterizing the frequency-current response curve, $I_{t h}$ is the threshold current and $I_{s}$ is the synaptic current and $V_{t h}$ is the voltage threshold, that is, the largest steady voltage at which the neuron can be maintained by a constant input. Without synaptic currents, $I_{s}=0$, the QIF neuron presents two distinct regimes. When $I_{t h}>0$ there are two fixed points. The stable one defines the resting state $V_{\text {rest }}$ of the neuron

$$
V_{\text {rest }}=V_{t h}-\sqrt{\frac{I_{t h}}{q}} .
$$

The unstable one is the threshold below which trajectories tend towards infinity in finite time that defines the spike time. When $I_{t h}<0$ the neuron fires regularly. This model represents the normal form of any type I neurons near the saddle-node bifurcation (Ermentrout, 1996; Ermentrout and Kopell, 1986) and is related to the so-called $\theta$ neuron (Ermentrout, 1996; Gutkin and Ermentrout, 1998). Since the quadratic integrate-and-fire neuron is expected to reproduce the characterisitcs of any type I neuron close to bifurcation, it has been widely used as a realistic neuron model (Hansel and Mato, 2001; Latham et al., 2000; Brunel and Latham, 2003; Fourcaud-Trocmé et al., 2003). The action potential is defined as a divergence of the voltage. In numerical simulations, one has to introduce a cutoff at a finite voltage $V_{\text {peak }}$. After a spike, the membrane potential is instantaneously reset to $V_{\text {reset }}$.

The synaptic current $I_{s}$ is induced by presynaptic spikes. An incoming spike at time $t^{f}$ triggers a postsynaptic current

$$
I_{s}(t)=w \delta\left(t-t^{f}\right),
$$

where $w$ is the synaptic weight of the synapse and $\delta$ is the dirac delta function. More realistic models use exponential currents

$$
I_{s}(t)=w \exp \left(-\left(t-t^{f}\right) / \tau_{s}\right),
$$


where $\tau_{s}$ is the synaptic time constant.

An event-driven simulation requires an analytical expression for the membrane potential or, at least, a closed form expression for the firing times. In many cases a given neuron in the network will not fire (its firing time is $+\infty$ ). In such situation the efficiency of the simulation is improved using a spike-test, i.e. an algorithm that checks quickly whether a neuron will fire. Note that the efficiency of the spike-test crucially depends on the overall activity of the network.

In this note, we describe a method to simulate exactly the quadratic integrateand-fire model (1) with the synaptic currents (3)-(4). The membrane potential is solved analytically and a spike-test is derived.

\section{Exact simulation}

We consider the dimensionless QIF model

$$
\frac{d V}{d t}=V^{2}-I_{t h}
$$

obtained from (1) by the change of variables $V \leftarrow q\left(V-V_{t h}\right) / C$ and $I_{t h} \leftarrow$ $q I_{t h} / C^{2}$ (for convenience we do not change the notations). We further consider an instantaneous synaptic current so that $V \rightarrow V+w$ when a spike is received, $V \rightarrow V_{\text {reset }}$ when a spike is emitted. We consider excitable neurons and we take a positive threshold current. In an interval with no spike (i.e., no incoming spike and no spike emitted by the neuron itself), equation (5) can be solved analytically with the separation-of-variables technique. The membrane potential evolves according to

$$
V(t)=-\sqrt{I_{t h}} \tanh \left(\sqrt{I_{t h}}(t+c)\right),
$$

where $c$ is a constant of integration given by the initial condition $V(0)=$ $-\sqrt{I_{t h}} \tanh \left(c \sqrt{I_{t h}}\right)$. For $V(0)<\sqrt{I_{t h}}$ the neuron goes back to its resting value and no spike is emitted. A spike is emitted for $V(0)>\sqrt{I_{t h}}$, i.e. for an initial value greater than the unstable fixed point of the neuron. In this case, we calculate ${ }^{1} c \sqrt{I_{t h}}=-\operatorname{atanh}\left(\sqrt{I_{t h}} / V(0)\right)+i \pi / 2$. Using $\tanh (x+i \pi / 2)=\operatorname{coth}(x)$ we have

$$
V(t)=-\sqrt{I_{t h}} \operatorname{coth}\left(\sqrt{I_{t h}} t-\operatorname{atanh}\left(\sqrt{I_{t h}} / V(0)\right)\right) .
$$

The firing time is obtained when $V(t)$ crosses $V_{\text {peak }}$ and is explicitly given using (7). In the limit $V_{\text {peak }} \rightarrow \infty$ the firing time is obtained equaling to zero the argument of the coth function and is simply given by

$$
t^{f}=\frac{1}{\sqrt{I_{t h}}} \operatorname{atanh} \frac{\sqrt{I_{t h}}}{V(0)} .
$$

An event-driven scheme for a network of QIF neurons with instantaneous coupling can be easily implemented using (8). The generalization of the event-based

\footnotetext{
${ }^{1}$ Depending on the initial condition $V(0)$, the constant $c$ can be a complex number
} 
simulation to more realistic synaptic currents is not trivial.

A network of QIF neurons with exponential current follows the equations

$$
\begin{aligned}
\frac{d V}{d t} & =V^{2}-I_{t h}+I_{s}, \\
\tau_{s} \frac{d I_{s}}{d t} & =-I_{s} .
\end{aligned}
$$

When a spike is received by a synapse, the current $I_{s}$ is instantaneously modified $I_{s} \rightarrow I_{s}+w$. Excitatory or inhibitory synapses are accounted through the sign of $w$ provided that they have an identical time constant $\tau_{s}$.

System (9-10) is analytically solvable in intervals with no spike and $V(t)$ is expressed using Bessel functions (see Appendix 5.1). Thus we can calculate very accurately the value of the membrane potential at any time.

It is possible to speed up the simulation if neurons that will not spike are detected. We will derive a test that quickly check whether a neuron will spike. Depending on the initial conditions $V(0), I_{s}(0)$ either a spike is emitted or the neuron goes back directly to its resting state. There is a curve $V^{*}\left(I_{s}\right)$ in the phase plane that defines the threshold curve, i.e. the neuron spikes if and only if its state is above this curve. The threshold curve is given by the stable variety of the unstable fixed point $\left(I_{s}, V\right)=\left(0, \sqrt{I_{t h}}\right)$ (see Fig. A).

When the spike test is positive we need to compute the firing time. This computation can be done very efficiently with standard methods of roots finding. These methods require an initial guess. We define $V^{+}$and $V^{-}$that evolve according to the following differential equations

$$
\begin{aligned}
& \frac{d V^{+}}{d t}=V_{\text {peak }}\left|V^{+}\right|-I_{t h}+I_{s}, \\
& \frac{d V^{-}}{d t}=2 \epsilon\left|V^{-}\right|-\epsilon^{2}-I_{t h}+I_{s},
\end{aligned}
$$

where $I_{s}$ follows (10). Here, we have used piecewise linear bounds of the quadratic nonlinearity (Fig. B, left). $\epsilon$ is an arbitrary parameter and we choose $\epsilon=1$. By construction we have, $\forall t \geq 0, V^{-}(t) \leq V(t) \leq V^{+}(t)$ (see Eqs. 9, 11, 12 and Fig. B, right). Since $V^{+}(t)$ and $V^{-}(t)$ are the membrane potentials of (piecewise) linear integrate-and-fire models we can calculate accurately the times of the threshold crossing (Brette, 2006). These times give an upper and a lower bound for the firing times of the QIF neuron that allow an efficient root finding. However when the network activity is high, the spike-test is most of the time positive and this method becomes time consuming. Moreover in the high activity regime, $V(0)$ is near the threshold curve and it is possible to derive a more accurate bound of the firing time. Another approximation as initial guess is obtained using the bounding QIF

$$
\frac{d V^{+}}{d t}=V^{+^{2}}-I_{t h}+\max \left(0, I_{s}(0)\right),
$$

that gives a lower bound for the firing time. Likewise, an upper bound can be obtained by using $\min \left(0, I_{s}(0)\right)$ in the equation above. The firing time of the bounding QIF model (13) can be calculated quickly and accuratly using an equation similar to (8). The comparison with the lower bound given by the bounding LIF is shown figure $\mathrm{C}$ for different values of the synaptic current. We 
calculate the relative error $E=\left|t^{f}-t_{b}^{f}\right| / t^{f}$ where $t^{f}$ is the firing time of the QIF and $t_{b}^{f}$ is the approximation given by either the bounding LIF (11) or the bounding QIF (13), respectively.

There exists a critical value $\tilde{V}_{0}\left(I_{s}(0)\right)$ of the membrane potential such that for $V>\tilde{V}_{0}$ the bounding QIF model gives a better approximation of the firing time. Note that for positive values of $V_{0}$ the bounding QIF model is always better. This case is achieved when the average activity is high, i.e. neurons in the network are most of the time near the threshold curve.

\section{Numerical results}

To illustrate our method, we simulate a network of $N$ identical QIF neurons receiving an external excitatory spike train modeled as a Poisson process with a constant rate of $10 \mathrm{kHz}^{2}$. The QIF neurons are coupled all-to-all through GABAergic inhibition. This scenario has been used to reproduce the oscillatory synchronization observed in early olfactory systems (Martinez, 2005; Ambard and Martinez, 2006). Numerical values of QIF neurons (Eq. 1) are taken from (Ambard and Martinez, 2006): $C=0.2 \mathrm{nF}, V_{\text {rest }}=-65 \mathrm{mV}, V_{t h}=-60.68 \mathrm{mV}$, $q=0.00643 \mathrm{mS} . V^{-1}, I_{t h}=0.12 \mathrm{nA}, V_{\text {peak }}=30 \mathrm{mV}$ and $V_{\text {reset }}=-70 \mathrm{mv}$. The synaptic time constant is $\tau_{s}=6 \mathrm{~ms}$ and the synaptic strength is $w=0.05 / \mathrm{N}$ for the inhibitory synapses and $w=5 \cdot 10^{-5}$ for the excitatory poissonian input. We wrote our event-driven simulator in $\mathrm{C}++$ based on the event-driven library MVASpike (Rochel and Martinez, 2003) available at

http://mvaspike.gforge.inria.fr. The Bessel functions needed for the exact computation of $V(t)$ (see appendix 5.1) were implemented by using the GNU Scientic Library (http://www.gnu.org/software/gsl). The threshold curve defining the spike test was very accurately fitted by a polynomial of degree three. Each time the spike test is positive, the firing time is computed by finding the root of $V(t)-V_{\text {peak }}$. This is accomplished by a few steps of a Newton-Raphson method starting from an initial guess obtained from the bounding QIF (Eq. 13). The precision achieved in the simulations is in the order of $10^{-7} \mathrm{~ms}$.

We first simulate a network of $N=100$ neurons. Initial conditions of the membrane potentials $V(0)$ are taken randomly between $V_{\text {reset }}$ and $V_{\text {peak }}$ so that the firing times of uncoupled neurons are uniformly distributed. This way, the network starts in a completely desynchronized state. When inhibition is blocked, individual neurons fire at $380 \mathrm{~Hz}$ on average (see Figure D, left for the spike raster of the first $100 \mathrm{~ms}$ of the simulation). The simulation time was about 20 min for $1 \mathrm{sec}$ of biological time on a portable PC running Linux at $1.86 \mathrm{GHz}$. This is a reasonnable time in regards to the large number of events encountered during the simulation (in the order of $10^{6}$ ). Moreover, we found that the spike test is positive $100 \%$ of the time so that any event from the poissonian input requires spike timing computation of the $N$ QIF neurons. When lateral inhibition is taken into account, the spike test is positive in $50 \%$ of the cases and neurons are synchronized with precise spiking activity at about $10 \mathrm{~Hz}$ (see Figure D, right). In such a case, all neurons fire almost at the same time.

Rather than searching for the next firing time within the entire population

\footnotetext{
${ }^{2}$ This mimics the interaction with 1000 excitatory presynaptic neurons firing at $10 \mathrm{~Hz}$ on average
} 
of neurons at each occurance of an event, it might be sufficient to perform the search among a random subset of $k$ neurons. This leads to a considerable speedup as $k<<N$, albeit at a cost of introducing errors in the simulation. A similar probabilistic speedup has been used in kernel methods (Scholkopf and Smola, 2002). In Appendix 5.3, it is shown that the error probability ${ }^{3}$ is given by $0.95^{k}$ for any $N$. Therefore, a subset of 59 neurons is already sufficient to get a low error probability. For $k=59$, the error probability equals 0.05 which is small enough to not perturb the synchronization as shown in Figure E, left for a network of $N=1000$ neurons coupled all-to-all ( $10^{6}$ synapses $)$. For comparison, Figure E, right shows the dynamics obtained by simulating the same network with $k=30$ (error probability of 0.21 ).

\section{Discussion}

Due to its simple dynamics the behavior exhibited by the leaky integrate-andfire model is limited. The QIF model has seen increasing interest in recent years, primarily because it reproduces the properties of detailed conductancebased neurons near the threshold. At low firing rate, the response of any type I neuron is described by the QIF neuron. In particular the QIF model reproduces the f-I curve of any type I neurons near the threshold. We have proposed a method to simulate the QIF neuron in an event driven way. We have presented simulations of the quadratic integrate-and-fire model with exponential synaptic currents.

In the numerical simulation of neural networks it is difficult to predict if errors on firing times will create numerical artefacts or will remain irrelevant. Event-driven methods are more precise than traditional time-stepping integration algorithms because the spike timings are calculated with an arbitrary desired precision. In general, implementation of an event-driven method require non-linear root-finding algorithms, for instance the Newton-Raphson method which converges exponentially. Thus calculations are exact in the sense that the limit of the computer accuracy is reached with few iterations of the method. The error done with Euler or Runge-Kutta methods is fundamentally different. It is inherent to these approximated methods and the precision is fixed with a polynomial decreasing with respect to the time-step. However when the number of events is large, event-driven schemes are time-consuming. This issue is implementation dependent and, in particular, depends on how the event queue is performed. Another important limitation of exact simulations is that it cannot be applied to any model. Implementation of exact simulations is possible if the membrane equations are analytically solvable. Extensions of our event driven scheme to other types of synaptic connections or spike-generating currents have to be studied case-by-case :

- A QIF neuron with exponential conductance-based synaptic currents follows

$$
\frac{d V}{d t}=V^{2}-I_{t h}+g\left(V-E_{s}\right)
$$

\footnotetext{
${ }^{3}$ Error probability is defined as the probability that the firing time computed with the probabilistic speedup is greater than the $5 \%$ first firing times of the entire population of neurons.
} 


$$
\tau_{s} \frac{d g}{d t}=-g
$$

where $g$ and $E_{s}$ is the synaptic conductance and the synaptic reversal potential, respectively. The membrane voltage is analytically solvable and can be written using Whittaker functions (see Appendix 5.2). Thus, an event-driven scheme, similar to the one proposed in this note, can be used to simulate exactly QIF neurons with synaptic conductances.

- The recently proposed exponential integrate-and-fire (EIF) model (FourcaudTrocmé et al., 2003; Brette and Gerstner, 2005) is given by

$$
\frac{d V}{d t}=-(V+E)+e^{V},
$$

where $V$ and $t$ are normalized variables and $E=\left(V_{T}-E_{l}\right) / \Delta_{T}$ with $V_{T}$ a threshold voltage, $E_{l}$ the leak potential, $\Delta_{T}$ the spike slope factor. Like the QIF model, the EIF model has a soft threshold. However the spike-generating current is no longer quadratic but exponential. It has been shown that the f-I curve of the EIF model matches the f-I curve of detailed conductance-based models for a range of input currents larger than for the QIF model. The EIF model with instantaneous current (3) has an implicit solution and the firing time is given by

$$
t^{f}=\int_{V(0)}^{V_{p e a k}} \frac{d u}{-(u+E)+e^{u}},
$$

for $V(0)$ greater than the unstable fixed point of (14). As for the QIF neuron, $V_{\text {peak }}$ controls the shape of the spikes. It is possible to use precalculated tables with an arbitrary precision for the integral for an eventdriven simulation of the EIF model. We do not know if the EIF model with exponential currents is analytically tractable or if an implicit solution can be found.

\section{Appendices}

\subsection{The QIF with exponential synaptic current}

The QIF neuron with an exponential synaptic current can be rewritten as a nonlinear and nonautonomous differential equation

$$
\frac{d V}{d t}=V^{2}-I_{t h}+I_{s}(0) e^{-t / \tau_{s}}
$$

The first step is to transform (15) into a linear ODE. We use the change of variables

$$
V(t)=-\frac{1}{y(t)} \frac{d y}{d t},
$$

to obtain

$$
\frac{d^{2} y}{d t^{2}}=\left(-I_{t h}+I_{s}(0) e^{-t / \tau_{s}}\right) y(t)
$$


We use the change of variables $u(t)=2 \tau_{s} \sqrt{I_{s}(0)} e^{-t /\left(2 \tau_{s}\right)}$ which transforms the equation into

$$
u^{2} \frac{d^{2} y}{d u^{2}}+u \frac{d y}{d u}+\left(u^{2}-4 \tau_{s}^{2} I_{t h}\right) y=0 .
$$

Let $J_{\alpha}(x)$ and $Y_{\alpha}(x)$ be the two independant solutions of the so-called Bessel's equation

$$
u^{2} \frac{d^{2} y}{d u^{2}}+u \frac{d y}{d u}+\left(u^{2}-\alpha^{2}\right) y=0,
$$

where $\alpha=-2 \tau_{s} \sqrt{I_{t h}}$. We have

$$
y(u)=J_{\alpha}(u)+c Y_{\alpha}(u),
$$

where $c$ is a constant defined by the initial condition. Using

$$
\frac{d J_{\alpha}(u)}{d u}=-J_{\alpha+1}(u)+\frac{\alpha}{u} J_{\alpha}(u),
$$

and a similar equation for $Y_{\alpha}$ we find that the membrane potential of the QIF neuron with exponential synaptic current (9-10) is analytically solvable. The membrane potential is given by

$$
V(t)=-\sqrt{I_{t h}}-\frac{u(t)}{2 \tau_{s}} \frac{J_{\alpha+1}+c Y_{\alpha+1}}{J_{\alpha}+c Y_{\alpha}} .
$$

where $J$ and $Y$ are Bessel functions evaluated at $u(t)=2 \tau_{s} \sqrt{I_{s}(0)} e^{-t /\left(2 \tau_{s}\right)}$. The Bessel functions can be expressed as a series of Gamma functions. Note that calculations with infinite series are not fundamentally different from calculating non-polynomial functions (like the exponential or logarithmic functions). Numerically, the first terms of the serie are necessary to reach a good precision. It is also possible to use precalculated tables to speed up the calculations.

\subsection{The QIF with exponential synaptic conductance}

The analytical integration of the QIF with exponential synaptic conductance proceeds along the same lines as the integration of the QIF with exponential currents. The analytical expression of the membrane potential uses Whittaker functions instead of Bessel functions. More precisely, let $W_{\mu, \nu}(x)$ and $M_{\mu, \nu}(x)$, the Whittaker functions, be the solutions of the differential equation

$$
\frac{d^{2} y}{d x^{2}}+\left(-\frac{1}{4}+\frac{\mu}{x}+\frac{1 / 4-\nu^{2}}{x^{2}}\right) y=0 .
$$

The membrane potential of the QIF model with exponential synaptic conductance is given by

$$
\begin{aligned}
V(t)= & 1 /\left(c W_{\alpha, \beta}+M_{\alpha, \beta}\right) *\left(E_{s} M_{\alpha, \beta}+\left(\sqrt{I_{t h}}-E_{s}\right) M_{\alpha-1, \beta}+\ldots\right. \\
& \left.+c E_{s} W_{\alpha, \beta}+c \tau_{s}\left(E_{s}^{2}-I_{t h}\right) W_{\alpha-1, \beta}\right),
\end{aligned}
$$

where $W$ and $M$ are Whittaker functions evaluated at $u(t)=\tau_{s} I_{s}(0) e^{-t / \tau_{s}}$, $\alpha=1 / 2+\tau_{s} E_{s}, \beta=\tau_{s} \sqrt{I_{t h}}$ and $c$ is a constant defined by the initial condition. Whittaker functions have power series expansions that could be used to enhance the efficiency of the calculations. 


\subsection{The probabilistic speedup}

Advancing the event-driven simulation to the next firing time requires the computation of the firing times of the $N$ neurons. This can be very time-consuming as $N$ is large. In case of synchronization, the neurons fire almost at the same time and it might be sufficient to compute the firing times $t_{i}, i=1 \cdots k$, among a random subset of $k$ neurons and advance the simulation to the next firing time $\min \left(t_{i}\right)$ obtained from this subset. This leads to a considerable speedup as $k<<N$, albeit at a cost of introducing errors in the simulation. We define the error probability $p_{e}$ as the probability that $\min \left(t_{i}\right)$ is greater than the $5 \%$ first firing times of the entire population of neurons. We then have $p_{e}=P\left(\min \left(t_{i}\right)>t^{\star}\right)$ where $t^{\star}$ is the maximum of the $5 \%$ first firing times of the $N$ neurons. Considering the $t_{i}$ s as identically and independently distributed firing times, we have $p_{e}=\left(P\left(t_{i}>t^{\star}\right)\right)^{k}$. Moreover, $P\left(t_{i}<t^{\star}\right)=0.05$ because we have considered the $5 \%$ first firing neurons in the definition of $t^{\star}$, and thus $p_{e}=0.95^{k}$.

\section{Acknowledgments}

Research supported by the INRIA cooperative research initiative RDNR.

\section{References}

Ambard, M. and Martinez, D. (2006). Inhibitory control of spike timing precision. NeuroComputing, 70:200-205.

Berry, M. J., Warland, D. K., and Meister, M. (1997). The structure and precision of retinal spike trains. PNAS, 94:5411-5416.

Bi, G. and Poo, M. (1998). Synaptic modifications in cultured hippocampal neurons: dependence on spike timing, synaptic strength, and postsynaptic cell type. J. Neurosci., 18:10464-10472.

Brette, R. (2006). Exact simulation of integrate-and-fire models with synaptic conductances. Neural Comp., 18:2004-2027.

Brette, R. and Gerstner, W. (2005). Adaptative exponential integrate-and-fire model as an effective description of neuronal activity. J. Neurophysiol., 94:3637-3642.

Brunel, N. and Latham, P. (2003). Firing rate of the noisy quadratic integrateand-fire neuron. Neural Comp., 15:2281-2306.

Ermentrout, G. (1996). Type i membranes, phase resetting curves, and synchrony. Neural Comp., 6:979-1001.

Ermentrout, G. and Kopell, N. (1986). Parabolic bursting in an excitable system coupled with a slow oscillation. SIAM J. Appl. Math., 46:233-253.

Fourcaud-Trocmé, N., Hansel, D., van Vreeswijk, C., and Brunel, N. (2003). How spike generation mechanisms determine the neuronal response to fluctuating inputs. J. Neurosci., 23:11628-11640. 
Gerstner, W. and Kistler, W. (2002). Spiking neuron models. Cambridge university press.

Gutkin, B. and Ermentrout, G. (1998). Dynamics of membrane excitability determine interspike interval variability: a link between spike generation mechanisms and cortical spike train statistics. Neural Comp.., 10:10471065 .

Hansel, D. and Mato, G. (2001). Existence and stability of persistent states in large neuronal networks. Phys. Rev. Lett., 10:4175-4178.

Hansel, D., Mato, G., Meunier, C., and Neltner, L. (1998). On the numerical simulations of integrate-and-fire networks. Neural Comp., 10:467.

Izhikevich, E. (2003). Simple model of spiking neurons. IEEE Transactions on Neural Networks, 14:1569-1572.

Latham, P., Richmond, B., Nelson, P., and Nirenberg, S. (2000). Intrinsic dynamics in neuronal networks, i. theory. J. Neurophysiol., 83:808-827.

Mainen, Z. and Sejnowski, T. (1995). Reliability of spike timing in neocortical neurons. Science, 1503:268.

Makino, T. (2003). A discrete-event neural network simulator for general neuron models. Neural. Comput. and Applic., 11:210-223.

Martinez, D. (2005). Oscillatory synchronization requires precise and balanced feedback inhibition in a model of the insect antennal lobe. Neural Comp., $17: 2548-2570$.

Mattia, M. and Giudice, P. D. (2000). Efficient event-driven simulation of large networks of spiking neurons and dynamical synapses. Neural Comp., $12: 2305$.

Rochel, O. and Martinez, D. (2003). An event-driven framawork for the simulation of networks of spiking neurons. Proc. 11th European Symposium on Artificial Neural Networks.

Rudolph, M. and Destexhe, A. (2006). Analytical integrate-and-fire neuron models with conductance-based dynamics for event-driven simulation strategies. Neural Comp., 18:2305.

Scholkopf, B. and Smola, A. (2002). Learning with kernels. MIT Press.

Shelley, M. J. and Tao, L. (2001). Efficient and accurate time-stepping schemes for integrate-and-fire neuronal networks. J. Comp. Neurosci., 11:111-119.

Tonnelier, A. and Gerstner, W. (2003). Piecewise linear differential equations and integrate-and-fire neurons: insights from two-dimensional membrane models. Phys. Rev. E, 67.

VanRullen, R., Guyonneau, R., and Thorpe, S. J. (2005). Spike times make sense. Trends in Neurosciences, 28:1-4. 
Figure. A. The $\left(I_{s}, V\right)$-phase plane for the QIF neuron with exponential synaptic currents. The $V$-nullcline is shown (bell-shape curve). The stable manifold of the saddle-node point $\left(0, \sqrt{I_{t h}}\right)$ defines the threshold curve above which the neuron fires. Trajectories starting under the threshold curve tend toward the stable resting state $\left(0,-\sqrt{I_{t h}}\right)$.

B. (left) Piecewise linear sector bounds on the nonlinearity. The nonlinearity is shown in full line. The dashed lines show piecewise linear sector bounds. (right) Bounding of the membrane potential of the quadratic integrate-and-fire neuron using piecewise linear integrate-and-fire models. Parameters are $V_{\text {peak }}=3$, $I_{t h}=2$.

C. The relative error on the spike timing of the QIF as a function of the initial value of the membrane potential $V_{0}$ for different values of the initial synaptic current. Comparison is done between the approximation obtained by the bounding LIF (bLIF) and by the bounding QIF (bQIF). (left) $I_{s}(0)=5$. (middle) $I_{s}(0)=7$. (right) $I_{s}(0)=10$. Other parameters are those of $\mathrm{B}$.

D. Event-driven simulations of a network of QIF neurons ( $N=100$ neurons). (left) Spike trains produced without inhibition. (right) Spike trains produced with lateral inhibition ( $10^{4}$ synapses $)$.

E. Event-driven simulations of a fully connected network of QIF neurons $(N=$ 1000 neurons, $10^{6}$ synapses). (left) Simulation with probabilistic speedup ( $k=$ $59)$. (right) Simulation with probabilistic speedup $(k=30)$. 
A

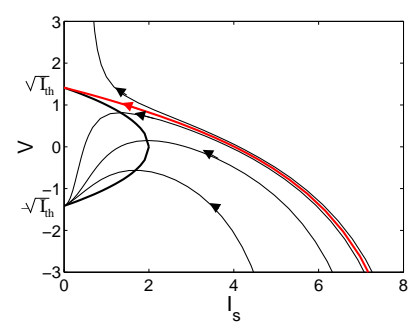

B
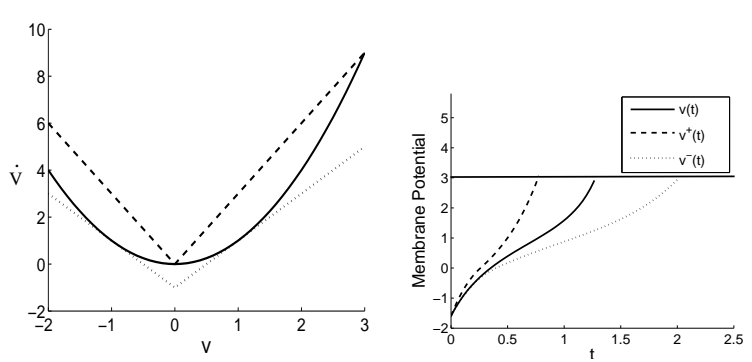

C
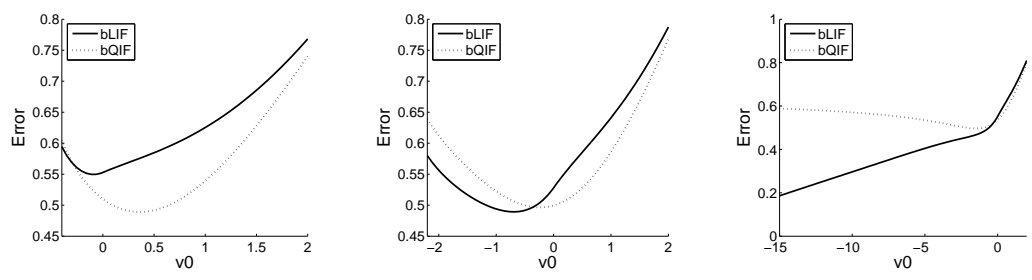

D
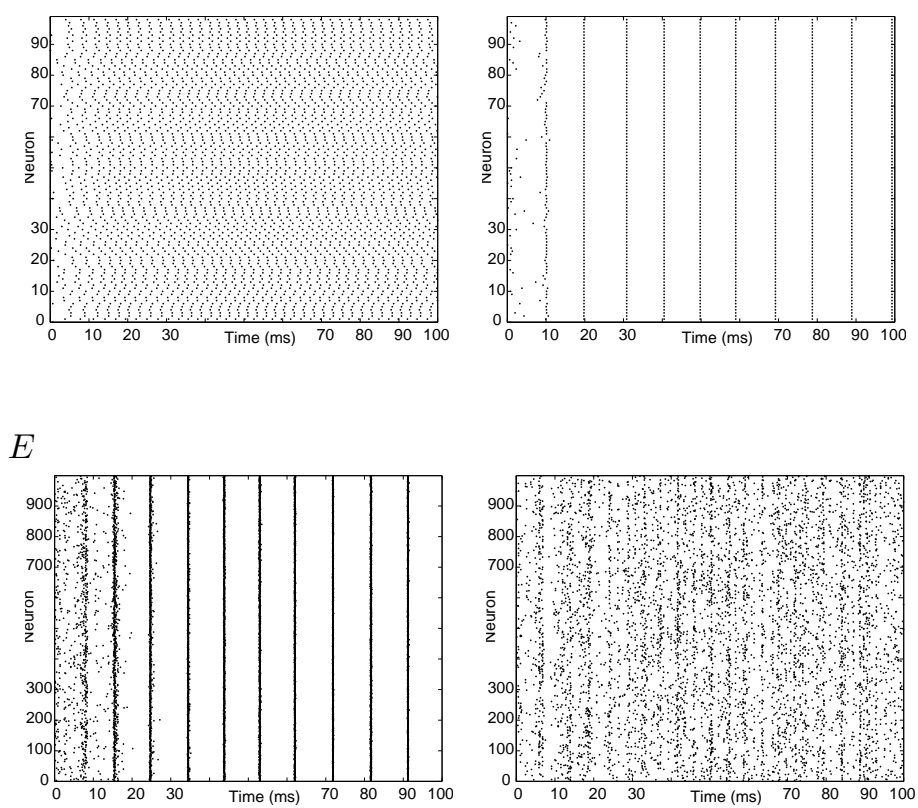